\title{
LOGISTICS CENTERS LOCATION
}

\author{
Vladimír Klapita ${ }^{1}$, Zuzana Švecová ${ }^{2}$ \\ Dept of Railway Transport, Faculty of Economics of Transport and Communication, \\ University of Žilina, Univerzitná ulica 8251/1, 01026 Žilina, Slovak Republic \\ E-mail:1Vadimir.Klapita@fpedas.utc.sk; ${ }^{2}$ Zuzana.Svecova@fpedas.utc.sk
}

Received 2 November 2005; accepted 4 January 2006

\begin{abstract}
It is fairly questionable to estimate future costs in the problems of strategic decision. The uncertainty may cause a resulting solution to be very inefficient considering current costs. In this report we try to find an approach which enables to determine a unique solution of a location problem at uncertain costs so that the solution is resistant to future changes. We deal with a sensitivity analysis and with a connection of an exact mathematical programming method and the theory of fuzzy sets.
\end{abstract}

Keywords: logistics, centre, optimal location of logistics centres, freight traffic, location problem, fuzzy sets, customers.

\section{Introduction}

In managerial experience we can find the problem of logistics centre optimal location. Location of such objects as manufactures, distributive and shopping centres, supply depots markedly affects the costs of material flows in creative logistic networks. The location of logistics centers is so much complicated because there is not only one logistic chain, but a whole distributive network.

Determination about the location or non-location of a logistics centre in some areas will affect the systems effectiveness for next several years. For finding the optimal solution it is possible to apply an exact method, but only at the known costs. When we solve location problems for most of them we have no real future costs, only their gross estimates. So it is necessary to deal with the approach of solving a location problem at uncertain costs.

This paper deals with a possible method of finding the optimal location of logistics centers at uncertain costs represented by fuzzy numbers.

\section{Formulation of a location problem}

A logistics centre can be set up only in some places from the finite set of possible locations which requires standby costs. In the system there are also costs of satisfying customer demands from some of located logistics centers, which depend on the quantity of requirements. The goal is to minimize complete costs of the system. So we have a difficult combinatorial problem of determination of a located logistics centers number.

There is securing freight traffic from one or more primary centres to customers in the distribution system. This freight traffic could be linear (without transshipments) or combined with transshipments in some centres called terminals which are often warehouses or buffer stocks. The structure of a distribution system is figured out by a set of primary centres, customers, terminals and flows of goods among them.

The location problem is a problem of optimal location of service centres on the given part of the transportation network.

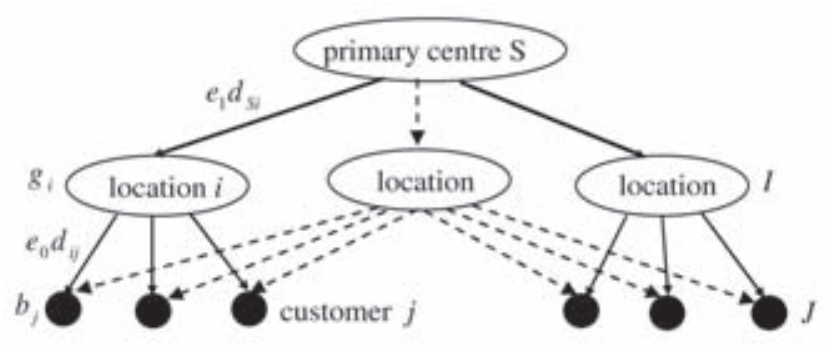

Fig 1. Two designs of a distribution system with transshipments 
The incapacitated location problem is conceived as follows:

The transportation network is given with customers in the nodes $j \in J$ and localities $i \in I$, in which it is possible to locate serve canters. Let's also assume that one centre located in the node from set $\mathrm{I}$ is able to serve all customers (see Fig 1).

The task is to minimize the complete costs which include standby costs $f_{i}$ paid for each location of the service centre in $i$ and variable costs $c_{i j}$ of demand satisfaction $b_{i j}$ of customer $j$ from terminal $i$. The variable costs for satisfying demand $b_{j}$ of customer $j \in J \quad c_{i j}=\left(e_{1} d_{S i}+e_{0} d_{i j}+g_{i}\right) b_{j}$ consist of charges $e_{1}$ for import from primary centre $S$ to terminal $i$, costs $g_{i}$ for transshipment in transshipment $i$ and charges $e_{0}$ for freight traffic from $i$ to customer $j$. The haul between the primary centre $\mathrm{S}$ and terminal $i$ is $d S_{j}$ and between terminal $i$ and customer $j$ it is $d_{i j}$. The condition is that all the customers have to be served, or more precisely have to be assigned to some of the located terminals.

Having introduced 0 - 1 variable $y_{i} \in\{0,1\}$ for each $i \in I$, which models the decision if the terminal is located at $i$ or not, and variable $z_{i j} \in\{0,1\}$ for each pair $i, j, \quad i \in I, \quad j \in J$, which assigns customer $j$ to terminal location $i$, we can set the following model of the complete cost minimization.

$$
\text { Minimize } f(y, z)=\sum_{i \in I} f_{i} y_{i}+\sum_{i \in I} \sum_{j \in J} c_{i j} z_{i j}
$$

where $c_{i j}=\left(e_{1} d_{s_{i}}+e_{0} d_{i j}+g_{i}\right) b_{j}$,

subject to $\sum_{i \in I} z_{i j}=1$ for $j \in J$,

$$
\begin{gathered}
z_{i j} \leq y_{i} \text { for } i \in I, j \in J, \\
z_{i j} \geq 0 \text { for } i \in I, j \in J, \\
y_{i} \in\{0,1\} \text { for } i \in I .
\end{gathered}
$$

In the model above the objective function (1) represents the complete costs of a distribution system. Constraints (2) ensure that each customer demand has to be satisfied from exactly one terminal location, constraints (3) force placement of a terminal at location i whenever a customer is assigned to terminal location $i$, constraints (4) ensure the location of terminal in every locality from which the demands of some customers are satisfied.

\section{Analysis of the existing approaches}

In strategic decision problems it is difficult to estimate future values of standby or/and variable costs. In this case the estimation of future costs is inaccurate. Considering confidential variables, which model determination about (un)location of terminals, the resulting solution can be economically inefficient in the view of the future costs. For example, the growth of $f_{i}$ or $e_{1}$ creates a change of system structure of location number and a change of customers assignment (see Fig 2). As a consequence, the estimation of expected costs by one numeric value is risky. Uncertain costs can be in such case described by an expectant interval of change of coefficient $f_{i}$ or $c_{i j}$, (but the uncertainty is too big) or by a fuzzy number which gives us more information about charges.

There are two approaches how to overcome the uncertainty.

First of them is a classical sensitivity analysis [1], which tells us how the optimal solution changes when some of the parameters have other value than the one which was calculated.

If uncertain parameter $f_{i}$ changes in interval $\left\langle f_{i}^{1}, f_{i}^{3}\right\rangle$, by dividing this interval into $m$ parts, we will have $m+1$ location problems with the known costs (6). But the result of sensitivity analysis is not a unique solution.

\section{Minimize}

$$
f(y, z)=\sum_{i \in I} f_{i} y_{i}+\sum_{i \in I} \sum_{j \in J} c_{i j} z_{i j} \quad f \in\left\langle f_{i}^{1}, f_{i}^{3}\right\rangle .
$$

Another approach uses the theory of fuzzy sets, where uncertain value $q$ is described by a possible interval and membership function $\mu_{q}$ (see Fig 3) - it is a power of applicability of a given element to $q$. This membership function has a triangular form.

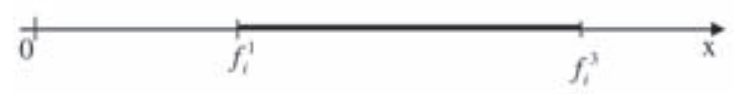

Fig 2. Interval of standby costs

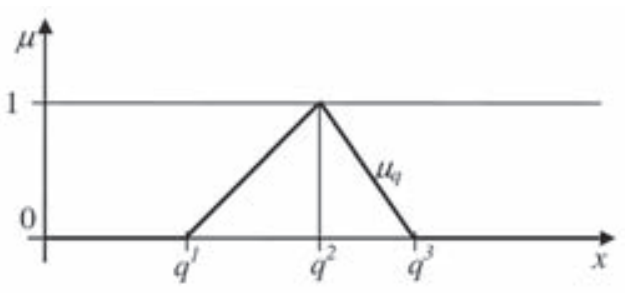

Fig 3. Membership function $\mu_{q}$

According to fuzzy arithmetic rules fuzzy numbers can be mutually added, subtracted and multiplied and divided by a real number without loss of the triangular form. When coefficients $q_{j}$ of objective function $F=q_{1} x_{1}+q_{2} x_{2}+\ldots+q_{n} x_{n}$ of a linear programming problem are triangular fuzzy numbers, then the value of the objective function for a given set of variable values $x=\left\langle x_{1}, x_{2}, \ldots, x_{n}\right\rangle$ is also a triangular fuzzy number 


$$
\begin{aligned}
& F(x)=\left\langle F^{1}(x), F^{2}(x), F^{3}(x)\right\rangle= \\
& =\left\langle\sum_{j=1}^{n} q_{j}^{1} x_{j}, \sum_{j=1}^{n} q_{j}^{2} x_{j}, \sum_{j=1}^{n} q_{j}^{3} x_{j},\right\rangle .
\end{aligned}
$$

The existing approach [2], which uses the theory of fuzzy sets looks for a solution for the given level of satisfaction $h$ which is given by an expert (see Fig 4). So we solve the original task, but with a changed objective function describing uncertain costs.

\section{Minimize}

$$
F^{*}(x)=F^{1}(x)+h\left(F^{2}(x)-F^{1}(x)\right) .
$$

The result of this method is concrete determination, but credibility of the associated result depends on expert's ability and his experience in determining a suitable level of satisfaction.

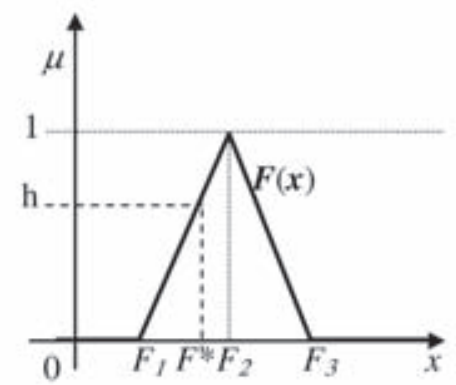

Fig 4. Membership function of fuzzy number $F$

\section{Concept of solving a location problem}

One method for finding concrete determination about the service centres location, which is not dependent on expert's ability, is the fuzzy algorithm [3]. This approach is based on introducing fuzzy set $F$, which expresses an assertion that "value of $F$ is small" with the membership function shown in Fig 5, where $F^{\text {min }}$ and $F^{\text {max }}$ denote respectively minimal values of $F^{1}(x)$ and $F^{2}(x)$ over a set of feasible solutions of the problem.

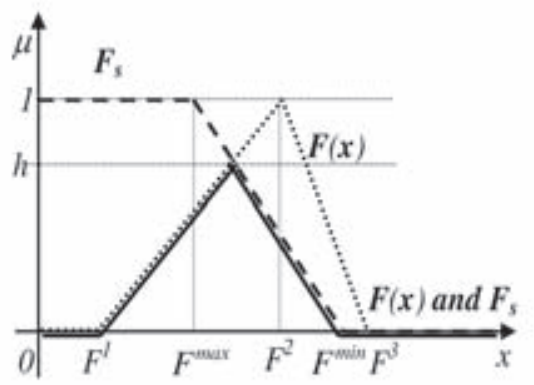

Fig 5. Membership function of fuzzy sets $F(x), F_{s}$ and their intersection $F(x)$ and $F_{s}$
In this approach we searched feasible solution $x^{*}$, for which the membership function of fuzzy set " $F(x)$ and $F_{s}$ " obtains maximal value $h$ (see Fig 5).

The maximal value $\mathrm{h}$ of the membership function of fuzzy set $F(x)$ and $F_{s}$ for given $x$ has to satisfy the following equality in the cases when $F^{1}(x) \leq F^{\max }$ holds.

$$
F^{1}(x)+\left(F^{2}(x)-F^{1}(x)\right) h=F^{\max }-\left(F^{\max }-F^{\min }\right) h
$$

In other cases $h$ can be set to zero. For the former case we get

$$
h(x)=\frac{F^{\max }-F^{1}(x)}{F^{2}(x)-F^{1}(x)+F^{\max }-F^{\min }}
$$

and we seek for $x^{*}$ maximizing $h(x)$, which is a nonlinear programming problem. The following numerical process [3] obtains an approximate solution of the problem.

1. Set $h$ to an initial positive value near zero.

2. Minimize the following objective function $F^{1}(x)+\left(F^{2}(x)-F^{1}(x)\right) h$ over the set of feasible $\mathrm{x}$ and denote $x *(h)$ the associated optimal solution.

3. Compute $h\left(x^{*}(h)\right)$ according to (10).

4.If $\left|h-h\left(x^{*}(h)\right)\right|<\varepsilon$ then stop else set $h=h(x *(h))$ and go to step 3 .

As it can be noticed in Fig 5 or derived from the expressions (9), the direct fuzzy approaches make use only of the left hand side of the membership function. It means that a part of fuzzy number from $F_{2}$ to $F_{3}$ is not taken into account (see Fig 6).

To overcome this weakness of the above mentioned fuzzy approaches, there is another fuzzy approach, which makes use of the membership function on its whole range. This approach resembles the way in which random coefficients are processed, when their distribution of probability is known. In this probabi-

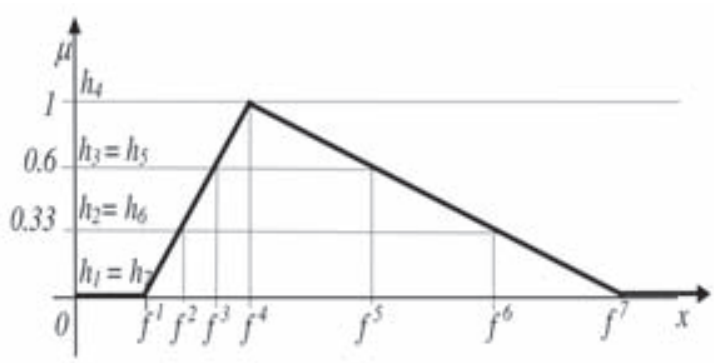

Fig 6. Level of satisfaction assignment to values of membership function 
listic-like approach the interval $[0,1]$ of possible values of the membership function is divided by real numbers of an arbitrary chosen finite set $H \subset[0,1]$. Then for each fuzzy coefficient $c$ from the location model values $c_{1}, c_{2}, \ldots, c_{r}$ are determined, so that constraint $\mu c\left(c_{\mathrm{k}}\right) \in H$ holds for $k=1,2, \ldots, r$. This is possible concerning the fact that the level of satisfaction of a fuzzy number centre is 1 .

Then we minimize the weighted sum function over feasible solutions $D$

$$
\min \left(\frac{\sum_{k=1}^{m+1} \sum_{i \in I} h_{k} f_{i}^{k} y_{i}}{\sum_{k=1}^{m+1} h_{k}}+\frac{\sum_{k=1}^{m+1} \sum_{i \in I} \sum_{j \in J} h_{k} c_{i j}^{k} z_{i j}}{\sum_{k=1}^{m+1} h_{k}}\right),
$$

subject to $(y, z) \in D$. The operating name of this method is weight 2 .

In the case we don't have more accurate information about uncertain costs, it means $h_{k}=1$ for $\forall k=1,2, \ldots, m+1$, the method is named minisum 2 .

If we use results of classical sensitivity analyses $(y, z) \in\left\{(y, z)^{1},(y, z)^{2}, \ldots,(y, z)^{m+1}\right\}$ in the weighted sum function and find for which of those results its value is minimal, it is the method minisum 1 . When we have nonzero weights, the method is named weights 1 .

To compare and verify both approaches, a branch and bound method was implemented and a software tool was built for sensitivity analysis and fuzzy processing of the location problem [4]. Functionality of the program was tested on 90 examples making use of the whole road system of Slovakia with 2906 dwelling places and 71 possible terminal locations. This way, in accordance with the primary source selection in 10 big towns of Slovakia 10 basic problems with predefined parameters $f, e_{1}, e_{0}$ were obtained. By three types of modification done independently with each of the three parameters, 90 benchmarks were obtained which were used in the experiments.

An average location number for the method weight 2 is $9 \pm 0,9$.

Average objective function value for method weight 2 is $32294726 \pm 3190392 S_{k}$.

\section{Conclusion}

The fuzzy algorithm computes a unique solution which is not dependent on expert's ability (like classical fuzzy method) and is resistant to future changes.

We have compared these approaches:

- $\quad$ sensitivity analysis and its usage by the methods minisum 1 , minisum 2 , weight 1 , weight 2 ;

- a classical fuzzy method,

- a fuzzy algorithm.

If the uncertain costs of a location problem are described by a triangular fuzzy number, both methods weight 2 and fuzzy algorithm are correct ways of finding the design of the distribution system. These methods give similar results (see Tables 1 and 2). There

Table 1. Differences of average locations number between weight 2 and other methods

\begin{tabular}{|l|c|}
\hline \multicolumn{1}{|c|}{ Method } & $\begin{array}{c}\text { Difference of average locations } \\
\text { number from } 9 \pm 0.9\end{array}$ \\
\hline weight 1 & $0.01 \Rightarrow 0.12 \%$ \\
\hline $\begin{array}{l}\text { classical fuzzy } \\
\text { method }\end{array}$ & $0.49 \Rightarrow 5.39 \%$ \\
\hline fuzzy algorithm & $1.09 \Rightarrow 12.0 \%$ \\
\hline minisum 1 & $0.06 \Rightarrow 0.61 \%$ \\
\hline minisum 2 & $0.06 \Rightarrow 0.61 \%$ \\
\hline
\end{tabular}

Table 2. Differences of average value of objective function between weight 2 and other methods

\begin{tabular}{|l|c|}
\hline \multicolumn{1}{|c|}{ Method } & $\begin{array}{c}\text { Differences of average objective } \\
\text { function value from } \\
32294726 \pm 3190392 \text { Sk }\end{array}$ \\
\hline weight 1 & $174298 \Rightarrow 0.54 \%$ \\
\hline $\begin{array}{l}\text { classical fuzzy } \\
\text { method }\end{array}$ & $660291 \Rightarrow 2.04 \%$ \\
\hline fuzzy algorithm & $1619143 \Rightarrow 5.01 \%$ \\
\hline minisum 1 & $172483 \Rightarrow 0.53 \%$ \\
\hline minisum 2 & $99119 \Rightarrow 0.31 \%$ \\
\hline
\end{tabular}

is the difference of $12 \%$ in the number of placed terminals (average is 9 terminals). We suggest performing both approaches and resulting design takes into account only if the results of these methods differ slightly. In an opposite case we suggest to perform an additional cost analysis and make the fuzzy cost more precise.

Comparing of the methods minisum 1 and minisum 2 with weight 2 is only the reference example, because these approaches don't take weight into accounts.

One of the program outputs is graphical representation of the solution, so a user can find out the stability of the optimal solution (see Fig 7).

It is possible to change the values of the chosen parameter and also the method - analysis of sensitivity, classical fuzzy approach or fuzzy algorithm. The results are: object time, value of objective function, optimal number of terminals and their names and also associated customers. 


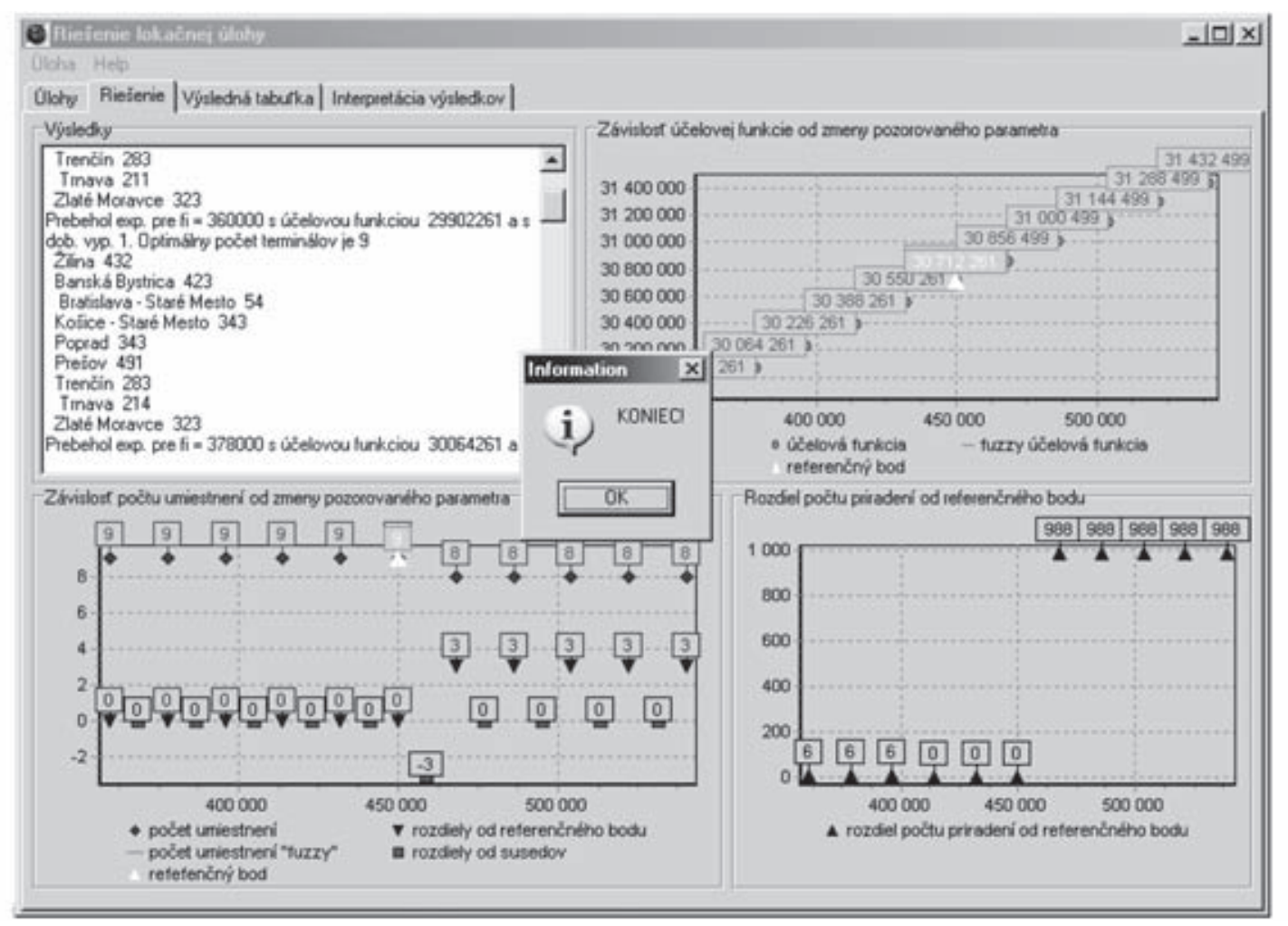

Fig. 7. Graphical output of the program

\section{References}

1. Janáček, J. Transport Network optimization (Optimalizace na dopravních sítích), ŽU-Žilina, 2002. 248 p. (in Slovak).

2. Teodorovič, D., Vukadinovič, K. Traffic control and transport planning: A fuzzy sets and neural networks approach, Boston, 1998. 387 p.

3. Janáček, J. Location problem under uncertain costs and charges. In: Proceedings of the 18th conference "Mathematical methods in economics", 13-15. 9. 2000, Prague, p. 85-90.

4. Švecová, Z. Location problem solving under uncertain costs. Diploma Thesis, Faculty of Management Science and Informatics, University of Žilina. 2003, 90 p. 